\title{
Supernova constraints on decaying vacuum cosmology
}

\author{
S. Carneiro, ${ }^{1,2, *, \dagger}$ C. Pigozzo, ${ }^{1,+}$ and H. A. Borges ${ }^{1, \S}$ \\ ${ }^{1}$ Instituto de Física, Universidade Federal da Bahia, 40210-340 Salvador-BA, Brasil \\ ${ }^{2}$ International Centre for Theoretical Physics, Trieste, Italy \\ J.S. Alcaniz ${ }^{3, \|}$ \\ ${ }^{3}$ Departamento de Astronomia, Observatório Nacional, 20921-400 Rio de Janeiro-RJ, Brasil
}

(Received 18 May 2006; published 28 July 2006)

\begin{abstract}
There is mounting observational evidence that the expansion of our Universe is undergoing a late-time acceleration. Among many proposals to describe this phenomenon, the cosmological constant $(\Lambda)$ seems to be the simplest and the most natural explanation. However, despite its observational successes, such a possibility exacerbates the well known $\Lambda$ problem, requiring a natural explanation for its small, but nonzero, value. In this paper we consider a cosmological scenario driven by a varying cosmological term, in which the vacuum energy density decays linearly with the Hubble parameter, $\Lambda \propto H$. We show that this $\Lambda(t) \mathrm{CDM}$ model is indistinguishable from the standard one $(\Lambda \mathrm{CDM})$ in that the early radiation phase is followed by a long dust-dominated era, and only recently the varying $\Lambda$ term becomes dominant, accelerating the cosmic expansion. In order to test the viability of this scenario, we have used the most recent type Ia supernova data, i.e., the High-Z SN Search Team and the Supernova Legacy Survey (SNLS) Collaboration data. In particular, for the SNLS sample we have found $0.27 \leq \Omega_{\mathrm{m}} \leq 0.37$ and $0.68 \leq$ $H_{0} \leq 0.72$ (at $2 \sigma$ ), which is in good agreement with the currently accepted estimates for these parameters.
\end{abstract}

DOI: 10.1103/PhysRevD.74.023532

PACS numbers: 98.80.Es, 95.35.+d, 98.62.Sb

\section{INTRODUCTION}

Over the last years, a considerable number of highquality observational data have transformed radically the field of cosmology. Results from distance measurements of Type Ia supernovae (SNe Ia) [1-3] combined with cosmic microwave background observations [4], dynamical estimates of the clustered matter [5], and age measurements of the oldest structures [6] seem to indicate that the simple picture provided by the standard cold dark matter scenario is not enough. These observations are usually explained by introducing a new hypothetical energy component with negative pressure - the so-called dark energy or quintessence (for recent reviews on this topic, see [7]). Besides its consequences on fundamental physics, if confirmed, the existence of this dark component would also provide a definitive piece of information connecting the inflationary flatness prediction with astronomical data.

On the other hand, from a purely theoretical viewpoint, the existence of a dark energy is related to an old problem of quantum field theories and theoretical cosmology, namely, the role of vacuum in the cosmic evolution [8]. Arguments of covariance and symmetry lead to an energymomentum tensor for the vacuum of the form $T_{\mu \nu}^{\Lambda}=$ $\Lambda g_{\mu \nu}$, where $\Lambda$ is a scalar function which, in spatially homogeneous and isotropic space-times, may be, at most, a function of time only. Therefore, the vacuum acts as a

\footnotetext{
*Associate member

†Electronic address: saulo@ fis.ufba.br

\#Electronic address: cpigozzo@ufba.br

${ }^{\S}$ Electronic address: humberto@ufba.br

"Electronic address: alcaniz@on.br
}

cosmological term, that is, as a perfect fluid with negative pressure given (in a comoving frame) by $p_{\Lambda}=-\rho_{\Lambda}=$ $-\Lambda$ [we work in units where $M_{P} \equiv(8 \pi G)^{-1 / 2}=c=1$ ].

Nevertheless, any tentative estimation of the vacuum energy density by quantum field theories in flat spacetime leads to a divergent result, and any natural cutoff we may choose to impose in those calculations leads to a vacuum contribution at least 40 orders of magnitude larger than the observed limits [9]. A possible way out of this trouble is to postulate some cancellation mechanism that leads to an exactly null vacuum contribution-the observed dark energy being due to a genuine cosmological constant or to other fields like quintessence or any other else. However, a more careful look at the problem of vacuum energy may suggest another possibility. The divergent result obtained by quantum field theories in flat space-time cannot, rigorously speaking, be used in the context of curved, expanding backgrounds. On the other hand, in Minkowski space-time the Einstein tensor is null, and, therefore, any vacuum contribution to the energymomentum tensor, divergent or not, should be canceled by a bare cosmological constant in Einstein equations. Now, if we could obtain the vacuum energy density in the expanding background, we should subtract the Minkowskian contribution, obtaining a "renormalized" vacuum density which would depend on the curvature, being very high for early times but decreasing as the Universe expands.

As is well known, the Bianchi identities $G_{\nu ; \mu}^{\mu}=0$ lead, via Einstein equations, to $T_{\nu ; \mu}^{\mu}=0$, which is an expression of energy-momentum conservation in the presence of the gravitational field [10]. In the Friedmann-Lemaittre- 
Robertson-Walker (FLRW) space-time, this equation leads to $\dot{\rho}+3 H(\rho+p)=0$, where $\rho$ and $p$ are the total energy density and pressure, respectively, while $H=\dot{a} / a$ is the Hubble parameter. By introducing the energy densities and pressures of vacuum and matter, one finds

$$
\dot{\rho}_{\gamma}+3 H\left(\rho_{\gamma}+p_{\gamma}\right)=-\dot{\Lambda} .
$$

The above equation is equivalent to a continuity equation for matter in the presence of a source $-\dot{\Lambda}$, meaning that the process of vacuum decay is concomitant to a process of matter production, a general feature of the vacuum state of any nonstationary space-time. We are probably far away from a definite theory of quantum vacuum in curved backgrounds or from the correspondent microscopic description of vacuum decay. Alternatively, we can consider effective, theoretically or empirically motivated, decaying laws for the vacuum density, exploring its effects by means of macroscopic equations like (1). Such an approach has an old history in the literature [11] and a renewed interest in recent years [12].

In this regard, a viable possibility has been proposed, with the vacuum density decaying as $\Lambda=\sigma H$, where $\sigma \approx$ $m_{\pi}^{3}$ has the order of the cube of the energy scale of the QCD vacuum condensation [13]. By using the observed values of $m_{\pi}$ and $H$, it is straightforward to verify that the above law provides a value for $\Lambda$ very close to the value presently observed. Naturally, the theoretical justification for this decaying law is based on some phenomenological hypothesis and, as such, needs to be verified from a fundamental theory viewpoint. However, the important aspect here is that it leads to cosmological solutions in agreement with the standard scenario for the evolution of the Universe, as explicitly shown in [14]. The above decaying law, for instance, leads to an early radiation-dominated phase where the vacuum term and the photon production are negligible, and where the scale factor and temperature evolve exactly as in the standard Friedmann solution. This phase is followed by a matter-dominated decelerating era, long enough to allow structure formation, and during which the vacuum density and matter production are dismissable until very recently. Finally, the Universe switches to an accelerated expansion driven by the vacuum, which tends asymptotically to a de Sitter solution. If we consider the present relative matter density (the only free parameter of the model, besides the Hubble constant) around 1/3, we also obtain the present age parameter $H_{0} t_{0} \approx 1$, which is in good accordance with current observations [6].

In this paper we are particularly interested in testing the viability of the above scenarios in light of the latest supernova (SNe Ia) data, as provided recently by Riess et al. [2] and by Astier et al. [3]. As is well known, these two SNe Ia samples constitute the compilation of best observations made so far and provide the most direct evidence for the observed late-time acceleration of the Universe. We also discuss other observational quantities, as the deceleration parameter $q$, the transition redshift $z_{\mathrm{T}}$ (at which the expansion switches from a decelerated to an accelerated phase), and the total expanding age of the Universe. In Sec. II we revise the main expressions and predictions of the cosmological solution with $\Lambda=\sigma H$. The observational quantities of the model are discussed in Sec. III. Section IV presents our SNe Ia analysis and a discussion on the observational constraints. In Sec. V we summarize our main conclusions.

\section{THE MODEL}

In the context of a FLRW space-time with null spatial curvature, the Einstein equations lead to the Friedmann equation

$$
\rho=\rho_{\gamma}+\Lambda=3 H^{2},
$$

which, together with (1), the equation of state for the matter fields $\left[p_{\gamma}=(\gamma-1) \rho_{\gamma}\right]$, and a decaying law for $\Lambda$, completely describes the evolution of the scale factor and densities. As discussed earlier, we will consider $\Lambda=$ $\sigma H$, so that by combining the above expressions we find

$$
2 \dot{H}+3 \gamma H^{2}-\sigma \gamma H=0 .
$$

By imposing the conditions $H>0$ and $\rho_{\gamma}>0$, we can also obtain the solution for the scale factor [14], i.e.,

$$
a(t)=C[\exp (\sigma \gamma t / 2)-1]^{2 / 3 \gamma},
$$

where $C$ is the first integration constant and the second one has been set equal to zero, in order to have $a=0$ at $t=0$. Note that the vacuum and matter densities are, respectively, given by $\Lambda=\sigma H$ and $\rho_{\gamma}=\rho-\Lambda=3 H^{2}-\sigma H$. Thus, by using Eq. (4), it is also possible to rewrite them as

$$
\rho_{\gamma}=\frac{\sigma^{2}}{3}\left(\frac{C}{a}\right)^{3 \gamma / 2}\left[1+\left(\frac{C}{a}\right)^{3 \gamma / 2}\right],
$$

and

$$
\Lambda=\frac{\sigma^{2}}{3}\left[1+\left(\frac{C}{a}\right)^{3 \gamma / 2}\right] .
$$

\section{A. Radiation-dominated era}

For the radiation epoch $(\gamma=4 / 3)$, Eq. (4) is given as

$$
a(t)=C[\exp (2 \sigma t / 3)-1]^{1 / 2},
$$

so that in the limit of small times $(\sigma t \ll 1)$, we have

$$
a \approx \sqrt{2 C^{2} \sigma t / 3},
$$

which is the same time dependence as in the standard scenario. Equations (5) and (6) reduce now to

$$
\rho_{r}=\frac{\sigma^{2} C^{4}}{3 a^{4}}+\frac{\sigma^{2} C^{2}}{3 a^{2}},
$$

and 


$$
\Lambda=\frac{\sigma^{2}}{3}+\frac{\sigma^{2} C^{2}}{3 a^{2}},
$$

while, in the limit $a \rightarrow 0$, we have

$$
\rho_{r}=\frac{\sigma^{2} C^{4}}{3 a^{4}}=\frac{3}{4 t^{2}},
$$

and

$$
\Lambda=\frac{\sigma^{2} C^{2}}{3 a^{2}}=\frac{\sigma}{2 t} .
$$

From the above expressions, it is straightforward to see that for small times the expansion is completely driven by the relativistic matter with its energy density scaling as $a^{-4}$.

\section{B. Matter-dominated era}

For the dust phase $(\gamma=1)$, Eq. (4) scales as

$$
a(t)=C[\exp (\sigma t / 2)-1]^{2 / 3},
$$

which means that for small times (compared to the present), it can be approximated by

$$
a(t)=C(\sigma t / 2)^{2 / 3} .
$$

Note that the above expression has the same time dependence as in the standard scenario. This, in other words, amounts to saying that the varying cosmological term starts dominating only very recently, which guarantees a large enough dust-dominated era.

For this matter-dominated epoch, Eqs. (5) and (6) read

$$
\rho_{m}=\frac{\sigma^{2} C^{3}}{3 a^{3}}+\frac{\sigma^{2} C^{3 / 2}}{3 a^{3 / 2}},
$$

and

$$
\Lambda=\frac{\sigma^{2}}{3}+\frac{\sigma^{2} C^{3 / 2}}{3 a^{3 / 2}} .
$$

The first term in (15) gives the usual scaling of nonrelativistic matter fields, whereas the second term is related to the production of matter at the expenses of the vacuum decay. Note that in the limit of large times $(\sigma t \gg 1)$, Eq. (13) leads to the de Sitter solution, i.e.,

$$
a(t)=C \exp (\sigma t / 3) .
$$

Note also [from (15) and (16)] that while $\rho_{m}$ tends to zero, $\Lambda$ tends to a genuine cosmological constant.

\section{OBSERVATIONAL QUANTITIES}

The Friedmann Eq. (2) for the dust-dominated epoch can be rewritten as

$$
H(z)=H_{0}\left[1-\Omega_{\mathrm{m}}+\Omega_{\mathrm{m}}(1+z)^{3 / 2}\right],
$$

where $\Omega_{\mathrm{m}}$ and $H_{0}$ stand for the current values of the relative matter density and Hubble parameters. From the above equation, it is straightforward to show that the deceleration parameter, defined as $q=-a \ddot{a} / \dot{a}^{2}$, now takes the following form:

$$
q(z)=\frac{\frac{3}{2} \Omega_{\mathrm{m}}(1+z)^{3 / 2}}{1-\Omega_{\mathrm{m}}+\Omega_{\mathrm{m}}(1+z)^{3 / 2}}-1,
$$

or, at the present time $(z=0)$,

$$
q(z=0)=\frac{3}{2} \Omega_{\mathrm{m}}-1 .
$$

Note that for any value of $\Omega_{\mathrm{m}}<2 / 3$ (as indicated by clustering and dynamical estimates [5]), the present-day cosmic expansion is accelerating, which seems to be in full agreement with current supernova observations [2,3] (see Sec. IV). From Eq. (19), it is also possible to obtain the transition redshift $z_{\mathrm{T}}$ at which the Universe switches from deceleration to acceleration, i.e.,

$$
z_{\mathrm{T}}=\left[2\left(\frac{1}{\Omega_{\mathrm{m}}}-1\right)\right]^{2 / 3}-1 .
$$

As one may anticipate, due to the process of matter production resulting from the vacuum decay, the transition redshift $z_{\mathrm{T}}$ in this model will be always higher than (but of the same order of) the transition redshift in a $\Lambda \mathrm{CDM}$ model for the same value of $\Omega_{\mathrm{m}}$. In reality, the cosmic acceleration in the presence of dust matter and a cosmological term is given by

$$
6 \frac{\ddot{a}}{a}=2 \Lambda-\rho_{m} .
$$

Therefore, the net effect of the additional terms in (15) and (16) is to increase the acceleration for a given value of $\rho_{m}$.

It is also possible to verify that the transition occurs soon after the first term in (15) is supplanted by the sum of the second term and $\Lambda$. In other words, the late-time acceleration starts just after the end of the dust matter epoch, which occurs at the redshift

$$
z^{*}=\left[(1+\sqrt{2})\left(\frac{1}{\Omega_{\mathrm{m}}}-1\right)\right]^{2 / 3}-1 .
$$

Finally, as shown in Ref. [14], the present age parameter can be expressed by

$$
H_{0} t_{0}=\frac{\frac{2}{3} \ln \left(\Omega_{\mathrm{m}}\right)}{\Omega_{\mathrm{m}}-1},
$$

which means that for the current accepted interval for the matter density parameter $\Omega_{\mathrm{m}}=0.30 \pm 0.05(2 \sigma)$ [5], one finds $H_{0} t_{0} \simeq 1.15 \pm 0.08$, i.e., in accordance with current age parameter estimates [6].

\section{SUPERNOVA CONSTRAINTS}

$\mathrm{SNe}$ Ia observations are certainly among the most remarkable findings of modern observational cosmology and provide the most direct evidence for the observed late-time cosmic acceleration. In this section we test the viability of the decaying vacuum scenario discussed above through a statistical analysis involving the most recent $\mathrm{SNe}$ Ia data, 
namely, the High-Z SN Search (HZS) Team [2] and the Supernova Legacy Survey (SNLS) Collaboration data [3].

\section{A. SNe Ia samples}

The total sample presented by the HZS Team consists of 186 events distributed over the redshift interval $0.01 \lesssim$ $z \lesssim 1.7$ and constitutes the compilation of the best observations made so far by them and by the Supernova Cosmology Project plus 16 new events observed by the Hubble Space Telescope. This total data set was divided into "high-confidence" (gold) and "likely but not certain" (silver) subsets. Here, we will consider only the 157 events that constitute the so-called gold sample.

The current data from the SNLS collaboration correspond to the first-year results of its planned five-year survey. The sample includes 71 high $-z \mathrm{SNe}$ Ia in the redshift range $0.2 \lessgtr z \lesssim 1$ and 44 low- $z$ SNe Ia compiled from the literature but analyzed in the same manner as the high $-z$ sample. This data set is arguably (due to multiband, rolling search technique, and careful calibration) the best high $-z$ SNe Ia compilation to date, as indicated by the very tight scatter around the best fit in the Hubble diagram and a careful estimate of systematic uncertainties. Another important aspect to be emphasized on the SNLS data is that they seem to be in a better agreement with Wilkinson Microwave Anisotropy Probe results than the gold sample (see, e.g., [15] for a discussion). In what follows we briefly outline the main assumptions for our analysis (see also [16] for some recent SNe Ia analysis).

\section{B. Statistical analysis}

The predicted distance modulus for a supernova at redshift $z$, given a set of parameters $\mathbf{s}$, is

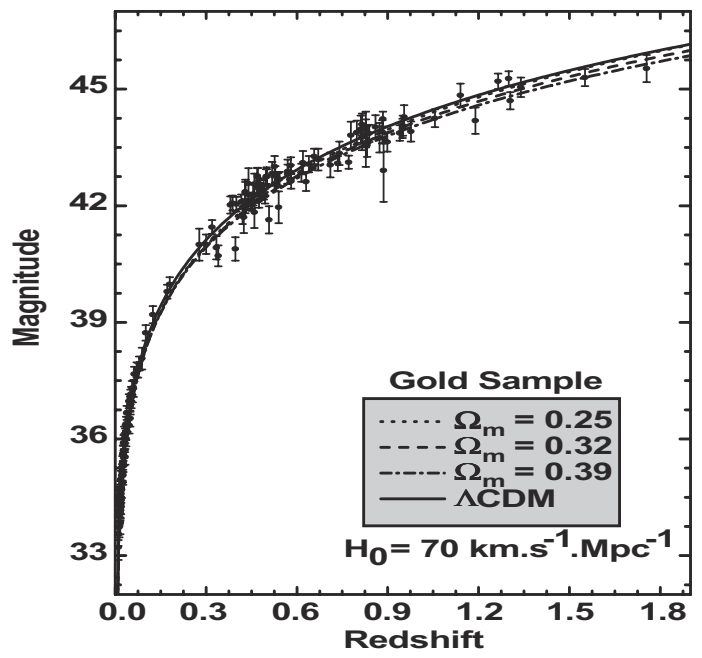

$$
\mu_{p}(z \mid \mathbf{s})=m-M=5 \log d_{L}+25,
$$

where $m$ and $M$ are, respectively, the apparent and absolute magnitudes, the complete set of parameters is $\mathbf{s} \equiv$ $\left(H_{0}, \Omega_{\mathrm{m}}\right)$, and $d_{L}$ stands for the luminosity distance (in units of megaparsecs),

$$
d_{L}=c(1+z) \int_{x^{\prime}}^{1} \frac{d x}{x^{2} H(x ; \mathbf{s})},
$$

where $x^{\prime}=\frac{R(t)}{R_{0}}=(1+z)^{-1}$ and $H(x ; \mathbf{s})$ the expression given by Eq. (18).

We estimated the best fit to the set of parameters $\mathbf{s}$ by using a $\chi^{2}$ statistics, with

$$
\chi^{2}=\sum_{i=1}^{N} \frac{\left[\mu_{p}^{i}(z \mid \mathbf{s})-\mu_{o}^{i}(z)\right]^{2}}{\sigma_{i}^{2}},
$$

where $N=157$ and 115 for gold and SNLS samples, respectively, $\mu_{p}^{i}(z \mid \mathbf{s})$ is given by Eq. (25), $\mu_{o}^{i}(z)$ is the extinction corrected distance modulus for a given $\mathrm{SNe}$ Ia at $z_{i}$, and $\sigma_{i}$ is the uncertainty in the individual distance moduli.

Figures 1(a) and 1(b) display the Hubble diagram for a fixed value of the Hubble parameter, $h(\equiv$ $\left.H_{0} / 100 \mathrm{Km} \cdot \mathrm{s}^{-1} \mathrm{Mpc}^{-1}\right)=0.7$, and some selected values of $\Omega_{\mathrm{m}}$. For the sake of comparison our current standard model, i.e., a flat $\Lambda$ CDM scenario with $\Omega_{\mathrm{m}}=0.27$, is also shown. Note that for the interval of $\Omega_{\mathrm{m}}$ considered, the predicted magnitude-redshift relation is very similar in both classes of scenarios, i.e., $\Lambda \mathrm{CDM}$ and $\Lambda(t) \mathrm{CDM}$. In Figs. 2(a) and 2(b) we show the results of our statistical analysis. Confidence regions (68.3\%, 95.4\%, and 99.7\%) are shown in the $\Omega_{\mathrm{m}}-h$ plane by considering the gold and SNLS samples, respectively. The best-fit parameters for the

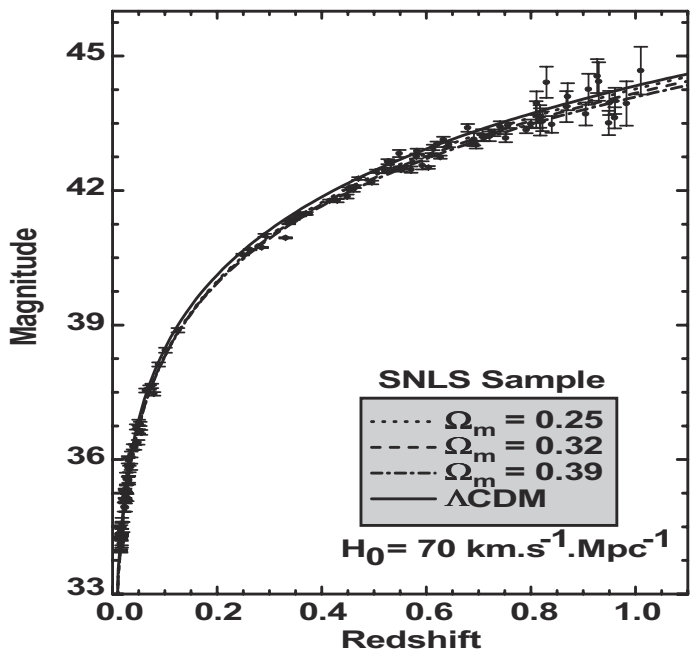

FIG. 1. Hubble diagram for 157 supernovae from HZS Team [Panel (a)] and 115 supernovae from SNLS Collaboration [Panel (b)]. As indicated in the figure, the curves correspond to a fixed value of the Hubble parameter, $h\left(\equiv H_{0} / 100 \mathrm{Km} \mathrm{s}^{-1} \mathrm{Mpc}^{-1}\right)=0.7$, and selected values of $\Omega_{\mathrm{m}}$. For the sake of comparison, the current standard cosmological model, i.e., a flat $\Lambda$ CDM scenario with $\Omega_{\mathrm{m}}=$ 0.27 , is also shown. 

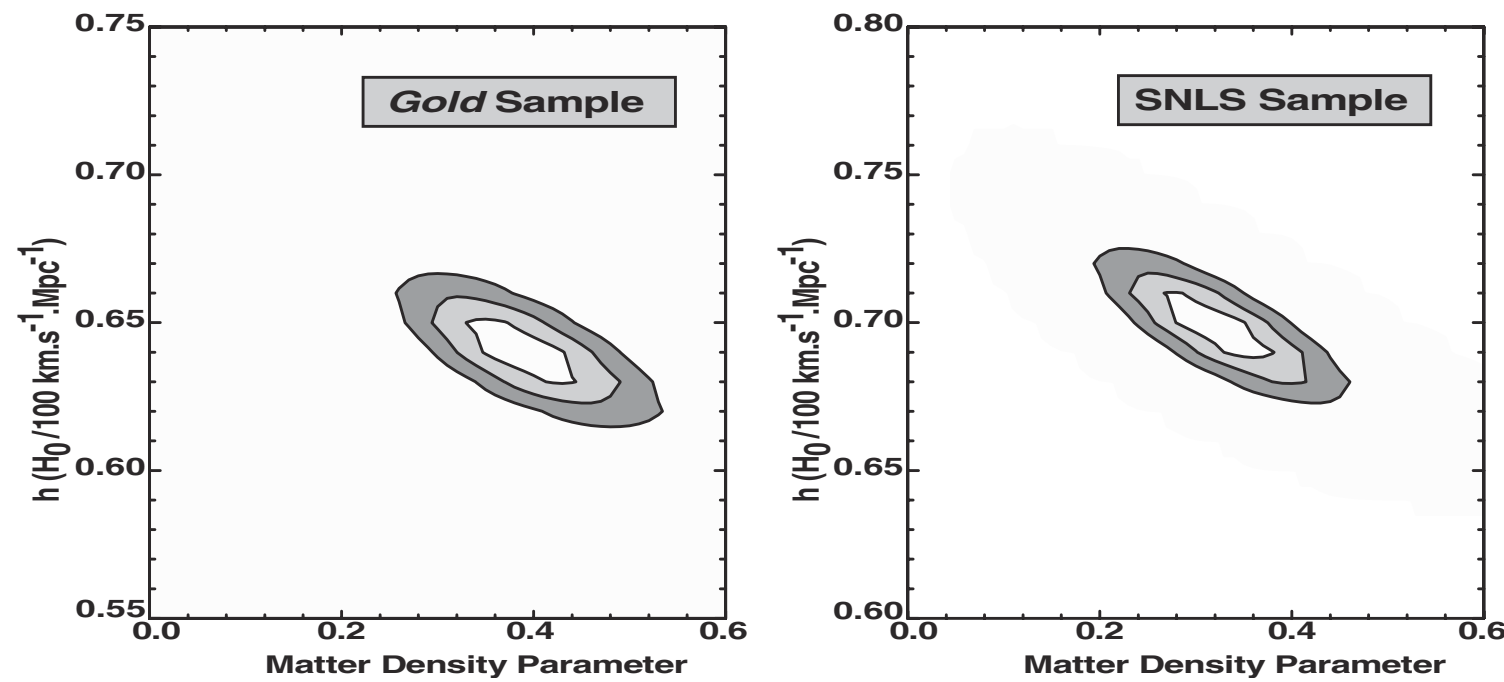

FIG. 2. The results of our statistical analysis. (a) Confidence regions in the $\Omega_{\mathrm{m}}-h$ plane for the gold sample of $157 \mathrm{SNe}$ Ia. The best-fit parameters for this analysis correspond to $\Omega_{\mathrm{m}}=0.39$ and $h=0.64$. (b) The same as in (a) for the SNLS sample of 115 SNe Ia. In this case, the best-fit model happens at $\Omega_{\mathrm{m}}=0.32$ and $h=0.70$, with $\chi_{r}^{2} \simeq 1.0$.

gold sample analysis are $\Omega_{\mathrm{m}}=0.39$ and $h=0.64$, with the reduced $\chi_{r}^{2} \equiv \chi_{\min }^{2} / \nu \simeq 1.14$ ( $\nu$ is defined as degrees of freedom). At $95 \%$ C.L. we also obtain the intervals $0.34 \leq \Omega_{\mathrm{m}} \leq 0.44$ and $0.62 \leq H_{0} \leq 0.66$. Note that these estimated values for the matter density parameter are considerably large, being only marginally compatible with the current accepted range, i.e., $\Omega_{\mathrm{m}}=0.30 \pm 0.05(2 \sigma)$ [5]. We also note that the above reduced value of $\chi^{2}$ is equal to the one found for the flat $\Lambda \mathrm{CDM}$ scenario and is slightly larger than the one obtained for the $\Lambda$ CDM model with arbitrary curvature $\left(\chi_{r}^{2} \simeq 1.12\right)$. For the SNLS analysis [Fig. 2(b)], the best-fit parameters are $\Omega_{\mathrm{m}}=0.32$ and $h=$ $0.7\left(\chi_{r}^{2} \simeq 1.0\right)$, which correspond to an accelerating universe with $q_{0} \simeq-0.52$, a total expanding age $t_{0} \simeq$ $15.7 \mathrm{Gyr}$, and a transition redshift $z_{\mathrm{T}} \simeq 1.62$ (with $z^{*} \simeq$ 1.97). At $95 \%$ C. L. we also obtain $0.27 \leq \Omega_{\mathrm{m}} \leq 0.37$ and $0.68 \leq H_{0} \leq 0.72$, which seem to be in better agreement with the current accepted values for both clustered matter density and Hubble parameters.

\section{CONCLUSIONS}

We have discussed a decaying vacuum scenario which is indistinguishable from the standard model with a genuine cosmological term in what concerns the general features of the predicted cosmic evolution. The early radiation phase in this $\Lambda(t) \mathrm{CDM}$ model is unaffected by the process of vacuum decay, as well as the physical phenomena taking place at early times (e.g., the primordial nucleosynthesis). The following era is dominated by dust for a long time, and only recently the varying cosmological term has become important.

Here, we have presented some quantitative results which clearly show that, even in the current stage of the Universe evolution, our decaying vacuum scenario is very similar to the standard one. We also have statistically tested the viability of the model by using the most recent $\mathrm{SNe}$ Ia observations, as given in Refs. [2,3]. For the so-called gold sample, we have found $0.34 \leq \Omega_{\mathrm{m}} \leq 0.44$ and $0.62 \leq$ $H_{0} \leq 0.66$ at $95.4 \%$ C.L., with the reduced $\chi_{r}^{2} \simeq 1.14$, which is equal to the one obtained in Ref. [2] for a flat $\Lambda$ CDM model. The SNLS data in turn provide $0.27 \leq$ $\Omega_{\mathrm{m}} \leq 0.37$ and $0.68 \leq H_{0} \leq 0.72$ (with $\chi_{r}^{2} \simeq 1.0$ ), which is in better agreement with the currently accepted estimates for both parameters. From the analysis presented above, we also noted that a more precise determination of the transition redshift $z_{\mathrm{T}}$ from upcoming $\mathrm{SNe}$ Ia data may be able to distinguish this scenario from the standard model since their predictions for this quantity are considerably different [see Eqs. (21) and (22)].

We also emphasize that an important observational aspect that deserves a careful investigation concerns the growth of density perturbations in the realm of this $\Lambda(t)$ CDM model. In this regard, a preliminary analysis indicates that the evolution of the matter contrast $\delta \rho / \rho$ shows no considerable difference relative to the standard $\Lambda \mathrm{CDM}$ case. A complete study on the formation of largescale structures in this class of scenarios will appear in a forthcoming communication.

Finally, it is worth observing that, from the theoretical viewpoint, it would be interesting to investigate possible relations between our approach and other quintessence models or modified gravitational theories recently discussed in the literature. For this purpose, we note that some authors have already pointed out a mathematical equivalence between dark energy and scalar-tensor or other forms of ideal fluid with inhomogeneous equations of state (see, e.g., [17]). 


\section{ACKNOWLEDGMENTS}

The authors are thankful to J. A. S. Lima for a useful discussion. C.P. and H. A. B. are supported by Capes. J.S.A. is supported by CNPq (Brazilian Agency), under
Grants No. 307860/2004-3 and No. 475835/2004-2 and by Fundação de Amparo à Pesquisa do Estado do Rio de Janeiro (FAPERJ), No. E-26/171.251/2004.
[1] S. Perlmutter et al., Astrophys. J. 517, 565 (1999).

[2] A. G. Riess et al., Astrophys. J. 607, 665 (2004).

[3] P. Astier et al., Astron. Astrophys. 447, 31 (2006).

[4] P. de Bernardis et al., Nature (London) 404, 955 (2000); S. Hanany et al., Astrophys. J. Lett. 545, L5 (2000); S. Padin et al., ibid. 549, L1 (2001); D. N. Spergel et al., Astrophys. J. Suppl. Ser. 148, 175 (2003).

[5] R. G. Calberg et al., Astrophys. J. 462, 32 (1996); A. Dekel, D. Burstein, and S. White, in Critical Dialogues in Cosmology, edited by N. Turok (World Scientific, Singapore, 1997); M. S. Turner, Astrophys. J. 576, L101 (2002); H. A. Feldman et al., Astrophys. J. 596, L131 (2003).

[6] B. M. S. Hansen et al., Astrophys. J. 574, L155 (2002).

[7] V. Sahni and A. A. Starobinsky, Int. J. Mod. Phys. D 9, 373 (2000); P. J. E. Peebles and B. Ratra, Rev. Mod. Phys. 75, 559 (2003); T. Padmanabhan, Phys. Rep. 380, 235 (2003); M. Tegmark et al., Phys. Rev. D 69, 103501 (2004); J. A. S. Lima, Braz. J. Phys. 34, 194 (2004); E. J. Copeland, M. Sami, and S. Tsujikawa, hep-th/0603057.

[8] S. Weinberg, Rev. Mod. Phys. 61, 1 (1989); P. J. E. Peebles and B. Ratra, Rev. Mod. Phys. 75, 559 (2003).

[9] This difference $\left[\simeq \mathcal{O}\left(10^{40}\right)\right]$ appears if we use a cutoff of the order of the energy scale of the QCD chiral phase transition, the latest of the cosmological vacuum transitions. For higher cutoffs, like the Planck mass $M_{P}$, the difference can be as big as 120 orders of magnitude.

[10] Note that, in principle, the general covariant structure of the Einstein field equations $\left(G_{\mu \nu}=T_{\mu \nu}^{M}+\Lambda g_{\mu \nu}\right)$ is not lost if one supposes $\Lambda$ to be a scalar function of coordinates $x^{\mu}$. Actually, if one considers the right-hand side of the above Einstein equations as a conserved, total energymomentum tensor $T_{\mu \nu}$, then no changes are needed in the general form of the Einstein-Hilbert action, although it would be necessary to know the actual interaction term between the matter and vacuum sectors.

[11] M. Bronstein, Phys. Z. Sowjetunion 3, 73 (1933); O. Bertolami, Nuovo Cimento Soc. Ital. Fis. B 93, 36 (1986); M. Özer and M. O. Taha, Phys. Lett. B 171, 363 (1986); Nucl. Phys. B287, 776 (1987); K. Freese et al., Nucl. Phys. B287, 797 (1987).

[12] W. Chen and Y.-S. Wu, Phys. Rev. D 41, 695 (1990); M. S. Berman, Phys. Rev. D 43, 1075 (1991); D. Pavón, Phys. Rev. D 43, 375 (1991); J. C. Carvalho, J. A. S. Lima, and I. Waga, Phys. Rev. D 46, 2404 (1992); A. I. Arbab and A. M. M. Abdel-Rahman, Phys. Rev. D 50, 7725 (1994); J. A. S. Lima and J. M.F. Maia, Phys. Rev. D 49, 5597 (1994); J. A. S. Lima and M. Trodden, Phys. Rev. D 53, 4280 (1996); J. M. Overduin and F. I. Cooperstock, Phys. Rev. D 58, 043506 (1998); J. M. Overduin, Astrophys. J.
517, L1 (1999); J. Shapiro and J. Sola, Phys. Lett. B 475, 236 (2000); M. V. John and K. B. Joseph, Phys. Rev. D 61, 087304 (2000); O. Bertolami and P. J. Martins, Phys. Rev. D 61, 064007 (2000); R. G. Vishwakarma, Gen. Relativ. Gravit. 33, 1973 (2001); A. S. Al-Rawaf, Mod. Phys. Lett. A 14, 633 (2001); M. K. Mak, J.A. Belinchon, and T. Harko, Int. J. Mod. Phys. D 11, 1265 (2002); I. L. Shapiro and J. Sola, J. High Energy Phys. 02 (2002) 006; M. R. Mbonye, Int. J. Mod. Phys. A 18, 811 (2003); S. Carneiro, Int. J. Mod. Phys. D 12, 1669 (2003); J.S. Alcaniz and J. M. F. Maia, Phys. Rev. D 67, 043502 (2003); I. L. Shapiro, J. Sola, C. Espana-Bonet, and P. RuizLapuente, Phys. Lett. B 574, 149 (2003); J. V. Cunha and R. C. Santos, Int. J. Mod. Phys. D 13, 1321 (2004); R. Opher and A. Pellison, Phys. Rev. D 70, 063529 (2004); R. Horvat, Phys. Rev. D 70, 087301 (2004); C. EspanaBonet et al. J. Cosmol. Astropart. Phys. 02 (2004) 006; P. Wang and X. Meng, Classical Quantum Gravity 22, 283 (2005); J. S. Alcaniz and J. A. S. Lima, Phys. Rev. D 72, 063516 (2005); S. Carneiro and J. A. S. Lima, Int. J. Mod. Phys. A 20, 2465 (2005); S. Carneiro, Int. J. Mod. Phys. D 14, 2201 (2005); S. Carneiro and A. E. Montenegro, Jr., Braz. J. Phys. 35, 1052 (2005); I. L. Shapiro, J. Sola, and H. Stefancic, J. Cosmol. Astropart. Phys. 01 (2005) 012; E. Elizalde, S. Nojiri, S. D. Odintsov, and P. Wang, Phys. Rev. D 71, 103504 (2005); J. Sola and H. Stefancic, Mod. Phys. Lett. A 21, 479 (2006); R. Aldrovandi, J. P. Beltrán Almeida, and J. G. Pereira, Gravitation Cosmol. 11, 277 (2005); F. Bauer, Classical Quantum Gravity 22, 3533 (2005); J.D. Barrow and T. Clifton, Phys. Rev. D 73, 103520 (2006).

[13] R. Schützhold, Phys. Rev. Lett. 89, 081302 (2002); Int. J. Mod. Phys. A 17, 4359 (2002).

[14] H. A. Borges and S. Carneiro, Gen. Relativ. Gravit. 37, 1385 (2005).

[15] H. K. Jassal, J.S. Bagla, and T. Padmanabhan, astro-ph/ 0601389.

[16] T. Padmanabhan and T. R. Choudhury, Mon. Not. R. Astron. Soc. 344, 823 (2003); P.T. Silva and O. Bertolami, Astrophys. J. 599, 829 (2003); Z.-H. Zhu and M.-K. Fujimoto, Astrophys. J. 585, 52 (2003); S. Nesseris and L. Perivolaropoulos, Phys. Rev. D 70, 043531 (2004); J.S. Alcaniz and N. Pires, Phys. Rev. D 70, 047303 (2004); J.S. Alcaniz, Phys. Rev. D 69, 083521 (2004); T. R. Choudhury and T. Padmanabhan, Astron. Astrophys. 429, 807 (2005); J. S. Alcaniz and Z.-H. Zhu, Phys. Rev. D 71, 083513 (2005); D. Rapetti, S. W. Allen, and J. Weller, Mon. Not. R. Astron. Soc. 360, 555 (2005); B. Feng, X. Wang, and X. Zhang, Phys. Lett. B 607, 35 (2005); R. Lazkoz, S. Nesseris, and L. Perivolaropoulos, J. Cosmol. 
Astropart. Phys. 11 (2005) 010; J. C. Fabris, S. V.B. Goncalves, and R. de Sa Ribeiro, astro-ph/0510779; Z.K. Guo, Z.-H. Zhu, J. S. Alcaniz, and Y.-Z. Zhang, astro$\mathrm{ph} / 0603632$.

[17] S. Capozziello, S. Nojiri, and S. D. Odintsov, Phys. Lett. B
634, 93 (2006); 632, 597 (2006); S. Nojiri and S.D. Odintsov, Phys. Rev. D 72, 023003 (2005); S. Capozziello, V.F. Cardone, E. Elizalde, S. Nojiri, and S. D. Odintsov, Phys. Rev. D 73, 043512 (2006). 